\title{
A Protocol for Imaging Axillary Lymph Nodes in Patients Undergoing Breast-Specific $\gamma$-Imaging
}

\author{
Elizabeth A. Jones, CNMT, RT(N), Trinh D. Phan, CNMT, RT(N), Nathalie M. Johnson, MD, \\ and Deborah A. Blanchard, DO \\ Department of Nuclear Medicine, Cancer Services, and Good Samaritan Breast Health Center Legacy Good Samaritan Hospital, \\ Portland, Oregon
}

\begin{abstract}
The development of small-field-of-view, breast-optimized, $\gamma$-camera designed for breast scintigraphy has resulted in improved breast lesion detection-particularly for lesions smaller than $1 \mathrm{~cm}$. However, unlike with the standard $\gamma$-camera, these images do not include the axilla within the field of view. Methods: Because of the effectiveness of breast scintigraphy using ${ }^{99 m} \mathrm{Tc}-$ sestamibi in the detection of axillary lymph node metastases, this article describes the development of an axillary imaging protocol for these small-field-of-view systems. Results: In addition, it describes how the improved resolution of these systems affects imaging of the axilla and reports observed, normal variants. Conclusion: Last, several example patient cases are discussed, describing both the impact and the limitations of this imaging protocol.
\end{abstract}

Key Words: breast; CNMT; molecular imaging; oncology; BSGI; axillary; lymph nodes

J Nucl Med Technol 2010; 38:28-31

DOI: 10.2967/jnmt.109.062711

\section{B} reast scintigraphy, achieved with general nuclear medicine cameras, has been performed for more than a decade; because of the large field of view, the standard lateral images include visualization of the breast, torso, and the axilla. Several studies have reported the detection of axillary node metastases from these images, and in many cases, these findings have been useful (Fig. 1). In 5 prospective masked-reader studies ( $n=814$ patients), the specificity of ${ }^{99 \mathrm{~m}} \mathrm{Tc}$-sestamibi for detecting metastatic breast cancer in axillary lymph nodes was $83 \%-100 \%$ (1-6). Furthermore, the high specificity of ${ }^{99 \mathrm{~m}} \mathrm{Tc}$-sestamibi in the detection of axillary metastases can be useful in the presurgical detection of primary node invasion for high-risk patients.

Axillary node involvement is a primary factor in prognosis and treatment planning (7), and although an intra-

\footnotetext{
Received Feb. 6, 2009; revision accepted Oct. 26, 2009.

For correspondence or reprints contact: Elizabeth A. Jones, Department of Nuclear Medicine, Legacy Good Samaritan Hospital, 1015 NW 22nd Ave., Portland, OR 97210.

E-mail: eljones@lhs.org

COPYRIGHT @ 2010 by the Society of Nuclear Medicine, Inc.
}

operative sentinel node procedure (the current standard of care) provides an excellent method for evaluating the lymph nodes, it can often result in multiple surgical procedures $(8)$. Because it is typically performed at the time of breast surgery, intraoperative pathologic evaluation of the node is associated with false-negative rates of up to $60 \%$; detailed pathology is conducted postoperatively, and patients with positive findings for sentinel node frequently return to surgery for axillary dissection (8). Although a negative ${ }^{99 \mathrm{~m}} \mathrm{Tc}$-sestamibi node study does not obviate a sentinel node procedure, positive ${ }^{99 \mathrm{~m}} \mathrm{Tc}$-sestamibi findings, given their high specificity, indicate the need for additional preoperative work-up, including ultrasound and, potentially, tissue sampling. If the presence of cancer can be determined preoperatively, the patient can undergo nodal dissection at the time of surgery and avoid the need for a sentinel node procedure or return to the operating room for delayed completion dissection.

Breast-specific $\gamma$-imaging, also known as BSGI, is a functional breast imaging study using a small-field-of-view $\gamma$-camera to mimic mammographic views and is becoming more available in the clinical setting. BSGI is often used after a cancer diagnosis to evaluate the extent of disease in the breast with the known cancer and evaluate the contralateral breast. At our institution, Zhou et al. reported that $10.9 \%$ of patients with known cancers had additional disease detected by BSGI (9). Our institution has incorporated axillary views at the time of BSGI to gain information on the status of the axillary lymph nodes to aid in surgical planning for these patients.

\section{MATERIALS AND METHODS}

In December 2006, Legacy Good Samaritan Hospital purchased the Dilon $6800 \gamma$-camera, to conduct breast scintigraphy at the breast imaging center. This study has also been referred to as BSGI and as molecular breast imaging in clinical literature. On the basis of the maneuverability of the small-field-of-view system and our institution's experience with mammography, the methods to image the breast were well understood. The $\gamma$-camera size and gantry design provided the flexibility required to emulate the same views obtained in mammography. Our nuclear medicine technologists spent several weeks learning the nuances of proper breast positioning and imaging from the mammography technologists to 


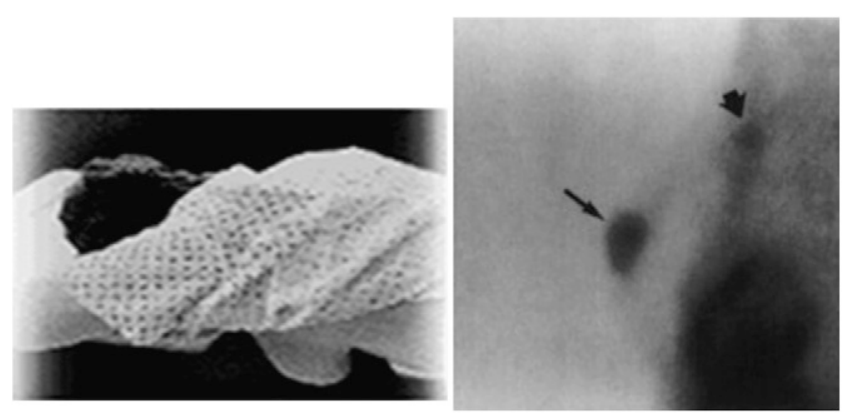

FIGURE 1. Photographs showing patient in prone breastpendant position (left) and example of standard $\gamma$-camera image of breast cancer with nodal involvement (right).

optimize breast positioning and to facilitate direct comparison between the modalities. Examples of these positions are shown in Figure 2. Each of these positions is described using the same nomenclature as for x-ray mammography. Although the breast images benefited from this interdisciplinary cross-training, neither specialty had a standard protocol for conducting imaging of the axillary nodes; thus, the optimal method for conducting imaging required investigation. The goal of this work was to develop procedural guidelines for acquiring images of the axilla using these specialized detectors.

\section{Injection Technique}

For breast imaging, ${ }^{99 \mathrm{~m}} \mathrm{Tc}$-sestamibi is typically injected into the venous system of the upper limb contralateral to the breast of concern. Most frequently, either the antecubital vein or a vein in the dorsal aspect of the hand is punctured. There are 2 complications with this technique that may cause artifacts in the images of the axilla-vascular trapping and subcutaneous extravasation. Vascular trapping occurs when a concentration of ${ }^{99 \mathrm{~m} T c-s e s t a m i b i}$ remains along the walls of the veins for more than $5 \mathrm{~min}$ after injection. Depending on the location of injection, this trapping may be visible on the axilla image as a linear region of increased concentration along either the basilic or the cephalic veins (Fig. 3). Subcutaneous extravasation occurs when fluid from the syringe leaks into the subcutaneous interstitial space between the skin and the vein. This fluid is cleared by the lymphatic system. In the case
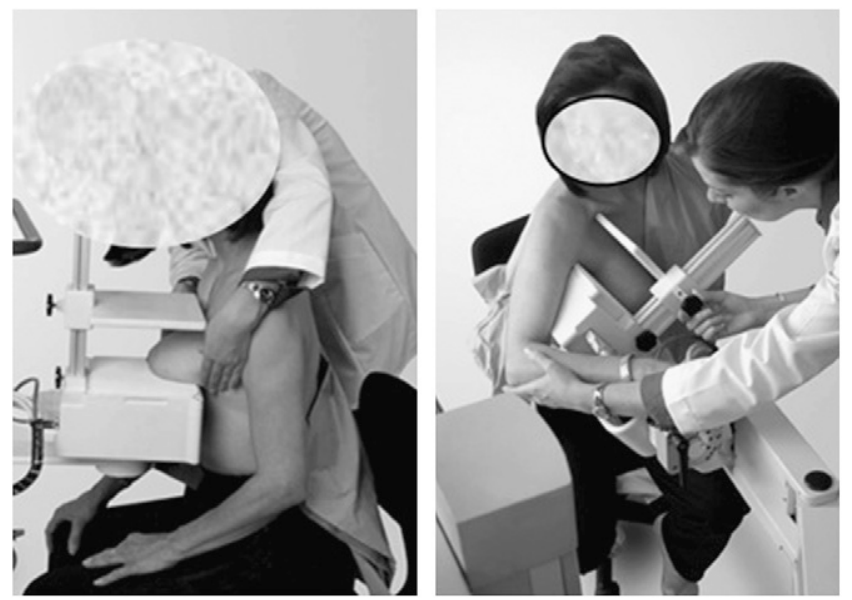

FIGURE 2. Photographs showing craniocaudal (left) and mediolateral oblique (right) positioning, typically noted as CC and MLO, respectively, in this BSGI study.

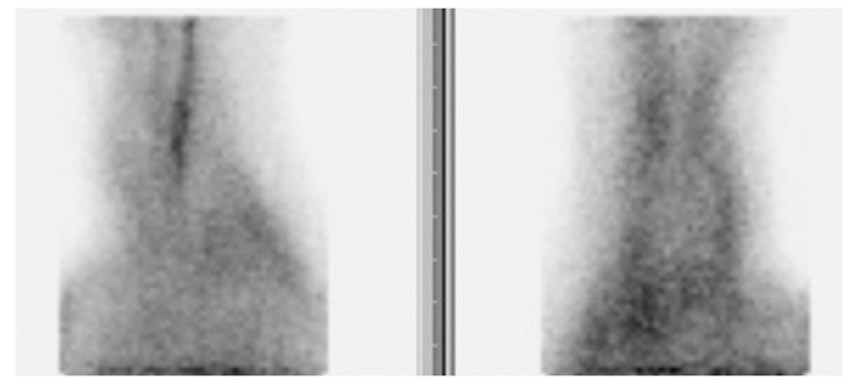

FIGURE 3. Bilateral axillary imaging study with venous trapping visualized in image on left (right axilla).

of ${ }^{99 m} \mathrm{Tc}$-sestamibi injections into the upper limb, this extravasation and subsequent lymphatic drainage can lead to concentrations of ${ }^{99 \mathrm{~m}} \mathrm{Tc}$-sestamibi in the axillary nodes, resulting in focal areas of uptake in the image of the axilla. If a focal enhancement is noted in the axilla of the arm of injection, an image of the injection site should be obtained. In cases of extravasation, there will be a diffuse intense distribution of ${ }^{99 \mathrm{~m}} \mathrm{Tc}$-sestamibi at the site of injection.

To reduce the probability of these complexities, the commonly used straight-stick injection technique was replaced by a method using a test, push, and flush technique. This technique requires an indwelling intravenous line, a syringe of ${ }^{99 \mathrm{~m}} \mathrm{Tc}$-sestamibi, a second syringe with $10-20 \mathrm{~mL}$ of saline, and a squeeze ball. Venous puncture is made and then verified by injecting $3-5 \mathrm{~mL}$ of saline through the line. Once the puncture is verified as patent, the ${ }^{99 m}$ Tc-sestamibi dose is administered, followed by the remaining saline as a flush. With the injection procedure complete, patients should then raise their arm to a level at which the elbow is above the ear and squeeze the ball in their hand for $1 \mathrm{~min}$. Although this technique does not eliminate the possibility of these artifacts, it has greatly reduced their frequency.

\section{Imaging}

The initial BSGI protocol included bilateral breast imaging with 2 views of each breast - craniocaudal and mediolateral oblique-with additional views added as requested by the interpreting physician. Under this initial protocol, axillary imaging was limited to unilateral images on the side of involvement in patients with a known breast cancer diagnosis. It was often difficult to interpret these images without a contralateral image for reference, and this frequently resulted in difficulty in reporting the results.

In addition, in these initial patients imaging of the axilla was conducted with the breast paddle attached to the detector. This paddle contains lead, which operates as a shield, helping to reduce imaging artifacts caused by ${ }^{99 \mathrm{~m}} \mathrm{Tc}$-sestamibi accumulation in nearby organs. For example, without the shield, the craniocaudal breast imaging view could contain artifacts from the distribution of ${ }^{99 \mathrm{~m}} \mathrm{Tc}$-sestamibi in glands of the head and neck. Figure 2 shows the typical positioning of the craniocaudal and mediolateral oblique views. Because of the proximity of these organs, it was suspected that these organs would also cause challenges in imaging the axilla. Initially, imaging was conducted with the paddle attached to the camera, but this positioning proved to be too difficult for most patients. Attempts to image without the paddle produced imaging artifacts that complicated image interpretation (Fig. 4). As a solution, the paddle was removed and a lead apron was draped across the patient's shoulder to shield the detector from uptake in the organs of the head (Fig. 5). 

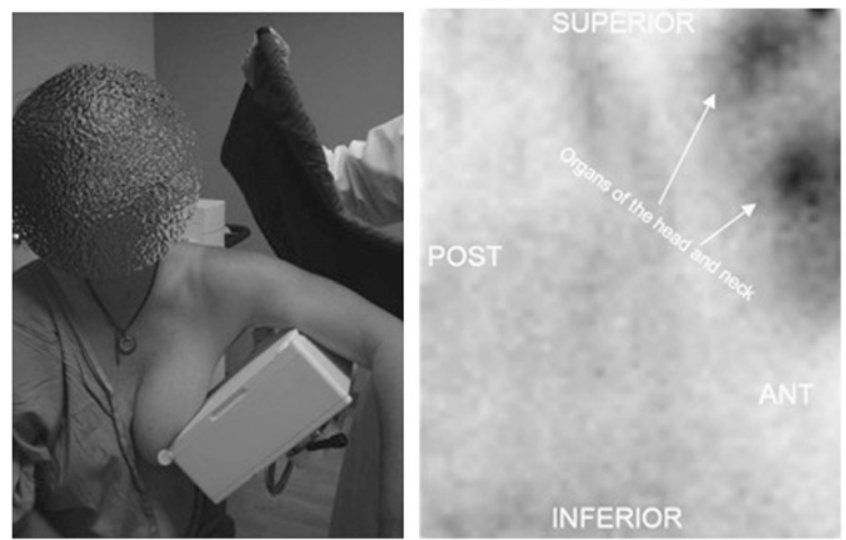

FIGURE 4. Photograph of axillary imaging position without paddle or shield (left). Resulting BSGI study shows uptake in head organs (right). POST $=$ posterior; ANT $=$ anterior.

Additionally, it is important to position the detector at an appropriate angle to avoid or minimize cardiac scatter when imaging the left side. This can be accomplished by angling the detector toward the head of the humerus.

\section{Acquisition Settings}

With the optimal imaging projection and method identified, we turned our attention to imaging time. Because of the low concentration of ${ }^{99 \mathrm{~m}} \mathrm{Tc}$-sestamibi in fatty tissue, the breast images obtained in a BSGI study are acquired for at least 5-10 min to obtain images over 150,000 counts (10). In comparison, the tissues in the axilla image contain a higher concentration of ${ }^{99 \mathrm{~m}} \mathrm{Tc}-$ sestamibi, and therefore a shorter imaging time is required for a 150,000-count image. Typically, $3 \mathrm{~min}$ per image is sufficient.

\section{RESULTS}

Over the course of 12 months through a methodic process of trial and error, the techniques described in this article were implemented. Each iteration improved the quality of the axillary images, and because of these improvements, our physicians have found greater benefit
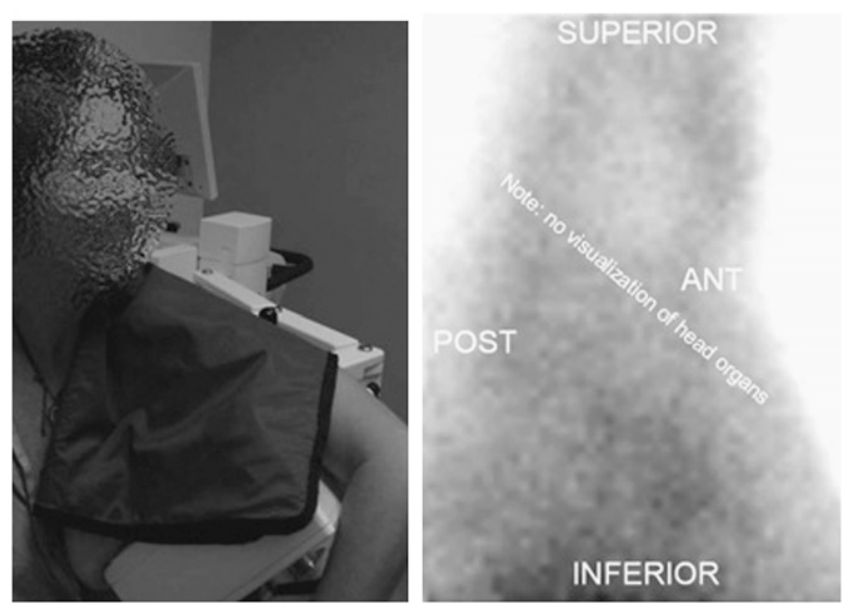

FIGURE 5. Photograph of same position as in Figure 4, with lead apron draped as shielding (left). BSGI study demonstrates elimination of head organs from image (right). POST = posterior; ANT = anterior. in imaging the axilla to assist in breast cancer staging. The presence of a focal abnormality in the axilla is highly indicative of nodal metastases but occasionally has been the result of a benign process. The causes of benign enhancements have been dose extravasation resulting in lymphatic trapping of ${ }^{99 \mathrm{~m}} \mathrm{Tc}$-sestamibi, lymphatic or other systemic infection, and inflammatory reactive nodes secondary to breast biopsy or surgery.

Although it is clear that these iterations have reduced the number of imaging artifacts and the complexity of interpreting these images, the impact has not yet been quantified in terms of sensitivity and specificity. A sentinel node dissection should still be conducted in patients with negative findings in the axillary images, because micrometastases in the nodes are not detectable with this imaging agent.

A case demonstrating the utility of this technique is provided in Figure 6. These images are from a patient who was referred for BSGI because of a newly diagnosed malignancy in the right breast. The right breast BSGI images show a large focal abnormality consistent with the known cancer, but no additional disease is detected in the right breast or axilla. However, the image of the left axilla shows a large bilobed area of intense uptake consistent with lymphadenopathy (Fig. 7). A subsequent preoperative ultrasound-guided biopsy confirmed the presence of adenocarcinoma of the breast.

\section{DISCUSSION}

At this institution, it has now become standard procedure to conduct bilateral imaging of the axilla in all patients

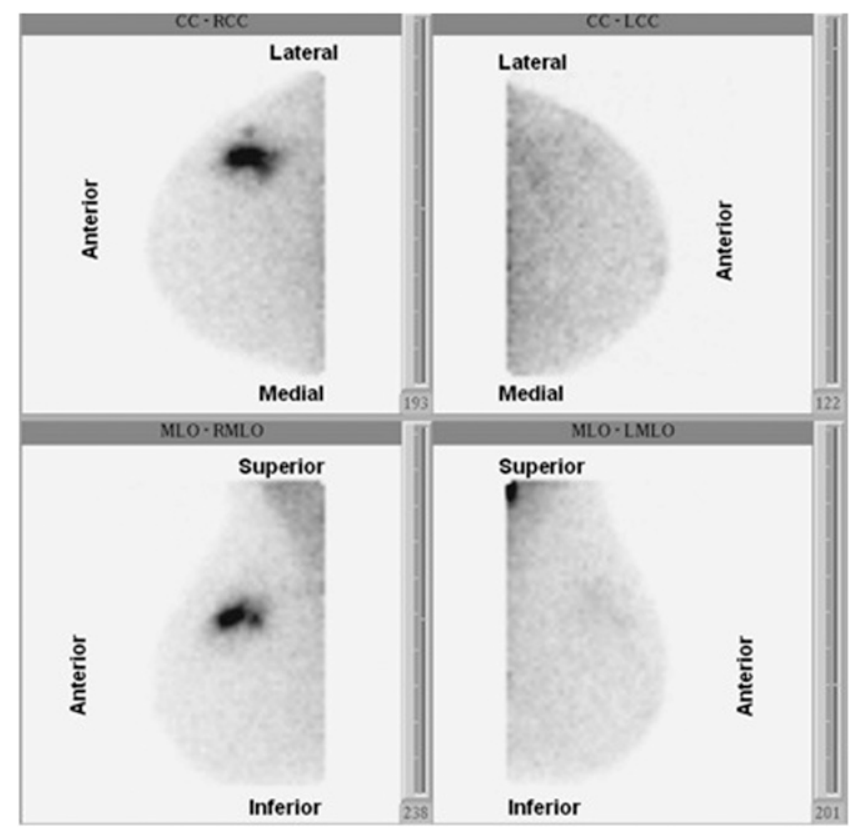

FIGURE 6. Bilateral breast BSGI study. Focal abnormality noted in both right craniocaudal (RCC) and right mediolateral oblique (RMLO) views. Left breast normal. LCC = left craniocaudal; LMLO = left mediolateral oblique. 

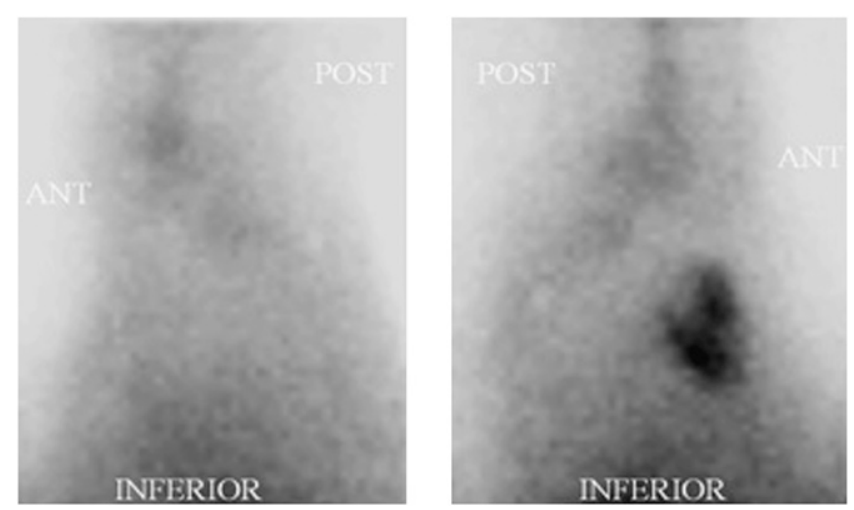

FIGURE 7. Bilateral axillary imaging study. Left axilla demonstrates grossly abnormal multifocal uptake. Right axilla is normal. POST $=$ posterior; ANT $=$ anterior.

undergoing BSGI. The patients tolerate this imaging procedure well, and it has become a valuable part of breast cancer diagnosis and treatment planning. In our experience, we have detected several malignant lesions missed by mammography, ultrasound, and sometimes MRI. This procedure has also proven useful for patients who have renal insufficiency, acute claustrophobia, ferromagnetic implants, obesity, or other factors limiting their ability to comply with an MRI order. The following procedural guidelines are the result of our experience, and they document the observations, modifications, and techniques we have determined to be useful in maximizing the effectiveness of axilla imaging in breast cancer patients.

\section{Procedural Guidelines}

- Place an intravenous line in the contralateral side if possible (a straight-stick technique is acceptable if intravenous placement is too difficult). Flush the line with saline to ensure intravenous patency.

- Flush the intravenous line with $20 \mathrm{~mL}$ of normal saline after the dose is administered.

- Remove the intravenous line, and have patients raise their arm over their head and squeeze/pump a small ball for 1 full minute. This is especially important when a straight-stick technique is performed.

- Have the patient expose the breast to be imaged through a gown opened in the front.

- Begin by imaging breasts. The order for imaging can vary; however, axillary imaging should begin at least 10 min after injection to decrease the chance of imaging vascular trapping.

- Remove the lead shield attached to the Dilon camera and set aside.

- Position the patient so the axilla is as close as possible to the camera face for the best resolution.

- Instruct the patient to hang her arm over the Dilon camera at a $90^{\circ}$ angle (Fig. 4, left).
- Place a lead apron across the shoulder and upper arm of the axilla that is being imaged (Fig. 5, left).

- Image the axilla for $180 \mathrm{~s}(3 \mathrm{~min})$ for best results. If the patient is unable to tolerate this time length, $120 \mathrm{~s}$ ( $2 \mathrm{~min}$ ) is adequate.

- Image the injection site for $60 \mathrm{~s}$, if there is any question of an extravasated dose.

- Use bilateral axillary imaging as a standard with all BSGI patients. This simply requires an extra 6 min of imaging per patient, with no increased radiation exposure and little, if any, discomfort for the patient.

\section{CONCLUSION}

BSGI has become an important diagnostic modality in the management of breast cancer patients. Although the standard and modified views of the breast are understood and benefit from the experience gained in mammography, there were no guidelines for optimizing imaging of nodes in the axilla. This work used an iterative process to develop guidelines to improve the quality of imaging the axillary nodes. Although several works have been published on the effectiveness of lymph node imaging using standard $\gamma$-cameras, further studies are required to determine the impact of these specialized detectors and the implementation of these guidelines. Future studies will determine what effect, if any, these specialized detectors will have in the sensitivity and specificity for axillary metastases in patients with primary breast cancer.

\section{REFERENCES}

1. Gommans GMM, van der Zant FM, van Dongen A, et al. ${ }^{99 m}$ Technetiumsestamibi scintimammography in nonpalpable breast lesions found on screening x-ray mammography. Eur J Surg Oncol. 2007;33:23-27.

2. Lumachi F, Tregnaghi A, Ferretti G, et al. Accuracy of ultrasonography and ${ }^{99 \mathrm{~m}} \mathrm{Tc}$-sestamibi scintimammography for assessing lymph node status in breast cancer patients: a prospective study. Eur J Surg Oncol. 2006;32:933-936.

3. Massardo T, Alonso O, Llamas-Ollier A, et al. Planar Tc99m-sestamibi scintimammography should be considered cautiously in the axillary evaluation of breast cancer protocols: results of an international multicenter trial. BMC Nucl Med. 2005;5:4.

4. Taillefer R, Robidoux A, Turpin S, et al. Metastatic axillary lymph node technetium-99m-MIBI imaging in primary breast cancer. J Nucl Med. 1998;39: $459-464$.

5. Lumachi F, Ferretti G, Povolato M, et al. Usefulness of 99m-Tc-sestamibi scintimammography in suspected breast cancer and in axillary lymph node metastases detection. Eur J Surg Oncol. 2001;27:256-259.

6. Clifford EJ, Lugo-Zamudio C. Scintimammography in the diagnosis of breast cancer. Am J Surg. 1996;172:483-486.

7. Escobar PF, Patrick RJ, Rybicki LA, Hicks D, Weng DE, Crowe JP. Prognostic significance of residual breast disease and axillary node involvement for patients who had primary induction chemotherapy for advanced breast cancer. Ann Surg Oncol. 2006;13:783-787.

8. Menes TS, Tartter PI, Mizrachi H, Smith SR, Estabrook A. Touch preparation or frozen section for intraoperative detection of sentinel lymph node metastases from breast cancer. Ann Surg Oncol. 2003;10:1166-1170.

9. Zhou M, Johnson N, Gruner S, et al. Clinical utility of breast specific gamma imaging for evaluating disease extent in the newly diagnosed breast cancer patient. Am J Surg. 2009;197:159-163.

10. Caldwell J, Mertens H, Linssen MC, van der Vusse GJ, Buell U, Kammermeier H. Uptake kinetics of technetium-99m-methoxyisobutylisonitrile and thallium-201 in adult rat heart endothelial and fibroblast-like cells in comparison to myocytes. J Nucl Med. 1992;33:102-107. 